\title{
Silicon photonic thermometer operating on multiple polarizations
}

\author{
Guan, Xiaowei; Wang, Xiaoyan; Frandsen, Lars Hagedorn
}

Published in:

2016 Conference on Lasers and Electro-Optics (CLEO)

Publication date:

2016

Document Version

Publisher's PDF, also known as Version of record

Link back to DTU Orbit

Citation (APA):

Guan, X., Wang, X., \& Frandsen, L. H. (2016). Silicon photonic thermometer operating on multiple polarizations. In 2016 Conference on Lasers and Electro-Optics (CLEO) (pp. 1-2)

\section{General rights}

Copyright and moral rights for the publications made accessible in the public portal are retained by the authors and/or other copyright owners and it is a condition of accessing publications that users recognise and abide by the legal requirements associated with these rights.

- Users may download and print one copy of any publication from the public portal for the purpose of private study or research.

- You may not further distribute the material or use it for any profit-making activity or commercial gain

- You may freely distribute the URL identifying the publication in the public portal

If you believe that this document breaches copyright please contact us providing details, and we will remove access to the work immediately and investigate your claim. 


\title{
Silicon Photonic Thermometer Operating on Multiple Polarizations
}

\author{
Xiaowei Guan*, Xiaoyan Wang, and Lars H. Frandsen \\ Department of Photonics Engineering, Technical University of Denmark, Ørsteds Plads Building 345A, 2800 Kgs. Lyngby, Denmark \\ *xgua@fotonik.dtu.dk
}

\begin{abstract}
A silicon photonics optical thermometer simultaneously operating on the multiple polarizations is designed and experimentally demonstrated. Measured sensitivities are $86 \mathrm{pm} /{ }^{\circ} \mathrm{C}$ and $48 \mathrm{pm} /{ }^{\circ} \mathrm{C}$ for the transverse-electric and transverse-magnetic polarizations, respectively. OCIS codes: (130.3120) Integrated optics devices; (130.6010) Sensors; (130.5440) Polarization-selective devices;
\end{abstract}

\section{Introduction}

Monitoring the ubiquitous variation of the ambient temperature is an important issue in many areas ranging from industrial manufacturing to life science [1]. Regarding the immunity to electromagnetic interference (EMI) inclined to happen in conventional electronic thermometers, temperature sensing utilizing optical methods is a promising alternative and is attracting much attention [2-4]. Compared with fiber-optic thermometers [2], silicon photonic thermometers have the superiorities of being more compact and CMOS compatible opening for mass manufacturing $[3,4]$. However, silicon waveguides inherently have a large birefringence [5], indicating that a thermometer designed for one polarization only will have a degraded performance due to instability in the polarization of the laser source or to polarization rotation along the circuit. Here, we demonstrate a novel silicon photonic thermometer, which can simultaneously operate on the transverse-electric (TE) and the transverse-magnetic (TM) polarizations.

\section{Principle and experimental results}

Figure 1(a) shows the waveguide configuration of the proposed silicon photonic thermometer, which consists of two identical subwavelength grating waveguides (SWGs) in a racetrack ring. The subwavelength grating part, shown as the inserted scanning electron microscopy (SEM) image in Fig. 1(a) is designed for TM mode to work in the air band while the TE mode is satisfying the Bragg condition of the grating. Thus, TM-polarized light will pass through the SWGs while TE-polarized light will be reflected. Such a SWG has previously been used as a TM-pass polarizer [6] and is, here, used as an in-line polarization beam splitter (PBS) consequently leaving TM-polarized light to resonate normally in the ring resonator and TE-polarized light to resonate between the two PBSs as in a Fabry-Pérot (FP) cavity. Figure 1(b) shows the calculated performances of the PBSs where both the transmission of the TM mode and the reflection of the TE mode can be larger than 0.8 from $1520 \mathrm{~nm}$ to $1600 \mathrm{~nm}$. Figure 1(c) shows the simulated electrical fields at $\lambda_{0}=1550 \mathrm{~nm}$ for the TE and the TM modes and clearly pictures the functionality of the PBSs. Here, we choose the height of silicon to be $205 \mathrm{~nm}$, the grating period to be $440 \mathrm{~nm}$, the number of periods to be 25 , a waveguide width of $500 \mathrm{~nm}$, and the width of the nano-bridge in the middle of the grating to be $150 \mathrm{~nm}$.
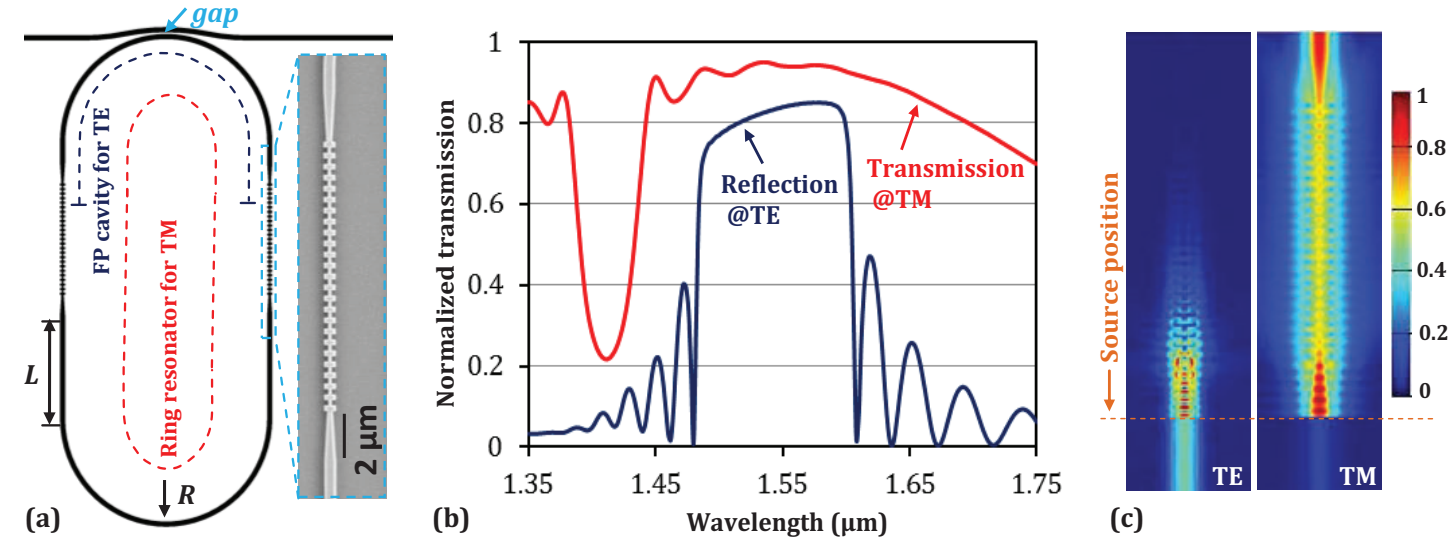

(c)

Fig. 1. (a) The proposed silicon photonic thermometer with subwavelength gratings acting as in-line PBSs. Inset shows the SEM image of the inline PBS. Here, $L=20 \mu \mathrm{m}$ and $R=20 \mu \mathrm{m}$. (b) Wavelength-dependence of the reflection of TE mode and the transmission of TM mode for the in-line PBS. (c) Light propagation for the TE and TM polarizations for the in-line PBS at the center wavelength of 1550nm. 
The designed thermometers were fabricated and measured at different temperatures controlled by a semiconductor cooler beneath the sample. Figures 2(a) and 2(b) show the measured transmission spectra at different temperatures for TM- and TE- polarized light, respectively. Here, the gap between the ring and the bus waveguide is $140 \mathrm{~nm}$. From the figures, one can see the free spectral range (FSR) for the TM polarization and the TE polarization is $3.98 \mathrm{~nm}$ and $3.88 \mathrm{~nm}$, respectively. Calculations show that for TM-polarized light the FSR would be $3.90 \mathrm{~nm}$ resonating in the ring and $4.90 \mathrm{~nm}$ assuming the TM mode to resonate in the FP cavity. Simultaneously, the calculations show that TE-polarized light would have a FSR of $3.85 \mathrm{~nm}$ in the FP cavity and a $2.93 \mathrm{~nm}$ FSR assuming the TE mode to resonate in the ring. Hence, the measured FSRs agree well with the calculated ones. Figure 2(c) shows the resonant wavelength shift with the temperature for the two polarizations. From the graph, the sensitivities of the proposed thermometer for TE and TM polarizations can be extracted as $86 \mathrm{pm} /{ }^{\circ} \mathrm{C}$ and $48 \mathrm{pm} /{ }^{\circ} \mathrm{C}$, respectively.
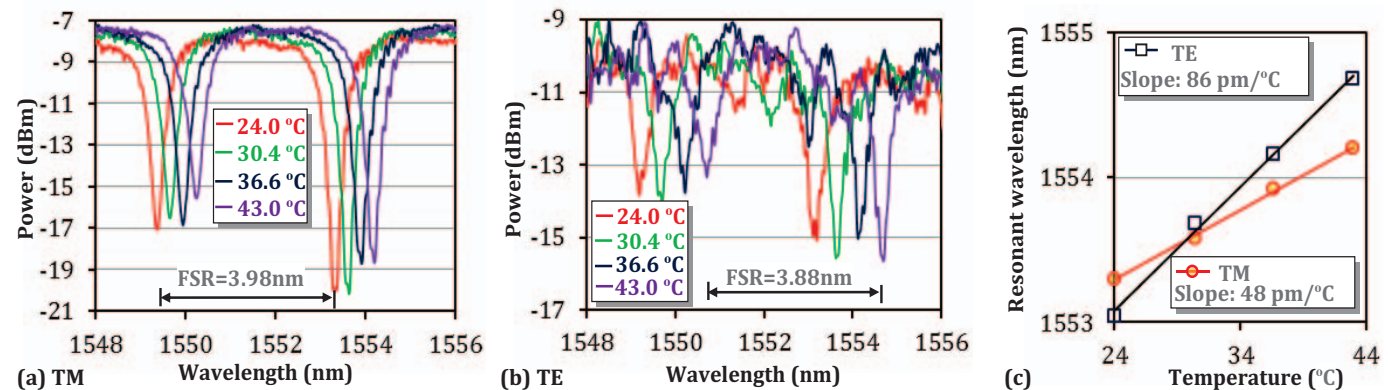

Fig. 2. Transmission spectra of a thermometer with the gap of 140nm for (a) TM- and (b) TE-polarized light. (c) The corresponding resonant wavelength shifts with temperature for (blue) TE- and (red) TM-polarized light.

Figures 2(a) and 2(b) show the extinction ratio (ER) of the thermometer is around $12 \mathrm{~dB}$ and $5 \mathrm{~dB}$ for TM- and TEpolarized light, respectively. To increase the ER for TE-polarized light, the gap between the ring and the bus waveguide can be lowered to couple more light into the ring. Figure 3 shows the transmission spectra for TM- and TE- polarized light for a thermometer with no gap. Clearly, the ER (at $24^{\circ} \mathrm{C}$ ) is improved to be larger than $9 \mathrm{~dB}$ for TE-polarized light and is kept to be around 10dB for TM-polarized light around the wavelength of $1550 \mathrm{~nm}$.

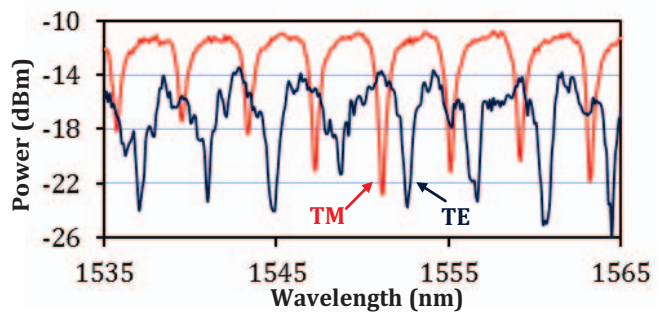

Fig. 3. Transmission spectra of a thermometer with no gap. Here, the temperature is $24^{\circ} \mathrm{C}$.

\section{Conclusion}

We have demonstrated a silicon photonic thermometer, which can simultaneously operate on the transverse-electric and transverse-magnetic polarizations to overcome the polarization instabilities stemming from large birefringence in on-chip thermometers. The use of in-line polarization beam splitters consisting of subwavelength grating waveguides in a racetrack ring resonator allows the two polarizations to resonate in different cavities. Measured temperature sensitivities of the resonant wavelengths for the two polarizations are $86 \mathrm{pm} /{ }^{\circ} \mathrm{C}$ and $48 \mathrm{pm} /{ }^{\circ} \mathrm{C}$. Our proposed design can also be applied to bio- or chemical-sensing for multiparameters monitoring since the operations of both polarizations can provide more sensing freedoms.

\section{References}

[1] J. Neupert et al., "Designing and using synthetic RNA thermometers for temperature-controlled gene expression in bacteria," Nat. Protoc. 4, $1262-1273$ (2009).

[2] H. Xu et al., "Ultra-sensitive chip-based photonic temperature sensor using ring resonator structures," OE 22, 3098-3104 (2014).

[3] Y. Rao et al., "In-fiber Bragg grating temperature sensor system for medical applications," JLT 15, 779-785 (1997).

[4] A. Irace et al., "All-silicon optical temperature sensor based on multi-mode interference," OE 11, 2807-2812 (2003).

[5] D. Dai et al., "Polarization management for silicon photonic integrated circuits," Laser Photon. Rev. 7, 303-328 (2013).

[6] X. Guan et al., "Low-loss ultracompact transverse-magnetic-pass polarizer with a silicon subwavelength grating waveguide," OL 39, 45144517 (2014).

\section{Acknowledgement}

This work was supported by the VILLUM foundation via the project 'Optical Nano-engineered Components for High-capacity Integrated silicon Photonics' (ONCHIP) 